\title{
Opinión
}

\section{Ley de embriaguez habitual en Honduras y su relación con el análisis}

\section{pericial toxicológico}

\section{Habitual Drunkenness Law in Honduras and its relationship with the toxicological expert analysis.}

\section{Dr. Josué Andrés Pineda Santos ${ }^{1^{*}}$}

${ }^{1}$ Laboratorio de Toxicología, Dirección de Medicina Forense, Honduras.

*Correspondencia a:

Josueaps 2011@hotmail.com

\section{Palabras claves}

Alcohol en sangre, Ley de embriaguez habitual, Escala Internacional prohibitiva, Cromatografía de Gases, Límite de detección, Honduras.

\section{Keywords}

Blood alcohol, Habitual drunkenness law, International prohibitive scale, Gas Chromatography, Detection limit, Honduras.

\section{Historia del artículo}

Recepción: febrero 2021

Aprobación: junio 2021

DOI:https://doi.org/10.5377/rcfh.v7 i1.11818

Citar como: Pineda Santos JA. Ley de embriaguez habitual en Honduras y su relación con el análisis pericial toxicológico. Rev. cienc. forenses Honduras. 2021; 7(1): 61-66. DOI: https://doi.org/10.5377/ rcfh.v7i1. $\underline{11818}$

Relaciones, actividades financieras y conflictos de interés: ninguno.

\section{https://orcid.org/0000-0002-0224-9382}

\section{RESUMEN}

A pesar que el número de accidentes de tránsito que involucran a conductores ebrios se ha disparado y que los legisladores tienen una gran presión para encontrar una solución razonable a la cuestión de la conducción bajo los efectos del alcohol especialmente en lo que respecta a los límites máximos permitidos, esa relación entre lo jurídico y lo científico, no siempre se concreta. El examen para la determinación de alcohol en muestras biológicas que se realiza en los laboratorios de Toxicología Forense de la Dirección de Medicina Forense fue validado y acreditado. Este proceso de validación permitió observar que el valor referido en la Ley de Penalización de la Embriaguez Habitual señala valores que podrían indicar un error en la aplicación de las unidades y de no ser así, dicho valor no sería significativo en cuanto a la determinación y cuantificación del etanol por la metodología actualmente utilizada en los laboratorios. La importancia del asesoramiento técnico científico para el uso adecuado de unidades y valores de referencia es indispensable para una interpretación objetiva de nuestra legislación y facilita el punto de unión entre la ciencia forense y la ciencia jurídica.

\section{ABSTRACT}

Despite the fact that the number of traffic accidents involving drunk drivers has skyrocketed, and that legislators are under great pressure to find a reasonable solution to the issue of driving under the influence, especially pressure to find a reasonable solution to
Rev. cienc. forenses Honduras, Volumen 7, N 1, Año 2021 Open Access
(C)Autor(es)
URL: http://www.bvs.hn/RCFH/html5/ CC-BY-NC 4.0 internacional 
the issue of driving under the influence, especially as regards the maximum limits allowed, that relationship between the legal and the scientific, is not always specified. The test for the determination of alcohol in biological samples that is carried out in the Forensic Toxicology laboratories of the Directorate of Forensic Medicine was validated and accredited. This validation process allowed to observe that the value referred to in the Law of Penalization of Habitual Drunkenness, point outs values that could indicate an error in the application of the units and if not, this value is not significant in terms of the determination and quantification of ethanol by the detection limit methodology currently used in laboratories. The importance of the advice of the scientific technician for the proper use of units and reference values is essential for an objective interpretation of our legislation and facilitates the meeting point in forensic science and legal science.

\section{INTRODUCCIÓN}

El estudio del consumo de alcohol y su impacto en la salud es complejo, no solo por la diversidad de formas de consumo existentes, sino por la gran heterogeneidad en su clasificación ${ }^{1}$, sin embargo, pese a que las bases científicas básicas de la relación del alcohol con los accidentes de tránsito se establecieron hace más de 70 años y la evidencia científica sobre el nivel de consumo de alcohol y su relación con el deterioro de las funciones psicomotoras es evidente ${ }^{2,3}$, el número de accidentes de tránsito que involucran a conductores ebrios se ha disparado; por lo que los legisladores tienen una gran presión para encontrar una solución razonable a la cuestión de la conducción bajo los efectos del alcohol ${ }^{4}$, especialmente a lo que respecta a los límites máximos en sangre permitidos. Por tal motivo, los países han establecido límites legales máximos de alcohol para los conductores, que van desde la tolerancia cero, hasta niveles diferenciados, de acuerdo al tipo de conductor.

\section{Límites máximos de etanol en sangre permitidos en la escala internacional}

En Estados Unidos, el límite legal varía entre $0,08 \mathrm{~g} / \mathrm{L}$ a $0,10 \mathrm{~g} / \mathrm{L}$ de alcohol en sangre, en la mayoría de los estados. Asimismo, en la mayoría de los países europeos el índice máximo de alcoholemia permitido es de $0,5 \mathrm{~g} / \mathrm{L}$ de alcohol en sangre, con un límite ligeramente más elevado, de $0,8 \mathrm{~g} / \mathrm{L}$, en Italia, Irlanda, Luxemburgo y el Reino Unido y un límite de 0,2 g/L en Suecia ${ }^{5}$, en Escocia recientemente se redujo el límite de $0,8 \mathrm{~g} / \mathrm{L}$ a $0,5 \mathrm{~g} / \mathrm{L}$ sin que este cambio se haya asociado a una reducción de los accidentes de tránsito ${ }^{6}$. En la Región Centroamericana y el Caribe los valores límites son diferenciados para Costa Rica y República 
Dominicana y de $0.5 \mathrm{~g} / \mathrm{L}$ para El Salvador, Nicaragua y Panamá ${ }^{7}$. En Honduras la Ley de Penalización de la Embriaguez Habitual, según decreto $\mathrm{N}^{\circ} 100-2000^{8}$, establece que el límite superior permitido en sangre es de 0,07 $\mathrm{mg} / 100 \mathrm{~mL}$ es decir $0,0007 \mathrm{~g} / \mathrm{L}$.

\section{Pruebas de detección de alcohol en sangre}

Según Silvia Miranda Arismendi, la definición médico legal de alcoholemia es: "la cantidad de alcohol que se detecta en la sangre de un individuo, si a su organismo hubiera ingresado alcohol o bebidas alcohólicas y que, por su acción sobre el sistema nervioso central, tendrá implicancia en un hecho judicial. La definición química de alcoholemia corresponde a la concentración de alcohol en la sangre" 9 .

En Honduras las muestras biológicas como sangre, orina y humor vítreo, se analizan en el Laboratorio Químico Toxicológico de la Dirección de Medicina Forense del Ministerio Publico. Para determinar y cuantificar los valores de etanol en sangre, se hace uso de la técnica de Cromatografía de Gases con doble detector FID, dicha técnica se caracteriza por su alta sensibilidad y permite la identificación de alcoholes, cetonas y aldehídos de bajo peso molecular. Esta técnica empleada en el Laboratorio de Toxicología ha sido validada y de manera posterior se logró su acreditación con la "ANSI National Accreditation Board", ANAB (https://anab.ansi.org/) con un alcance de análisis de determinación y cuantificación de etanol en sangre, con esta acreditación los resultados son acordes a los requerimientos de la norma ISO 17025. La ANAB realiza un proceso de evaluación continua de los laboratorios acreditados y verifica que se apliquen los métodos y protocolos adecuados de trabajo, que el personal cuente con la suficiente formación, experiencia y con los medios necesarios para ejecutar los análisis, como en el caso del Laboratorio de Toxicología de Medicina Forense de Honduras, con el ensayo de alcohol.

\section{Límite de detección mínimo en el análisis de alcohol en sangre y su relación con el nivel de alcohol en sangre legalmente permitido.}

El límite de detección mínimo se trata de la concentración mínima de analito que puede ser detectada e identificada y que puede diferenciarse del ruido de fondo, con un determinado grado de certeza estadística ${ }^{10}$. Para el caso, el límite de detección mínimo para la técnica de cromatografía de gases doble FID del Laboratorio Químico Toxicológico considerando el plan de validación del método para una concentración determinable y para cuantificable debe ser mayor o igual a $10 \mathrm{mg} / 100 \mathrm{ml}(0.1 \mathrm{~g} / \mathrm{L})$. Un valor menor a este límite únicamente puede ser detectado, más no interpretado, porque se podría asociar con 
otros elementos circunstanciales.

Este valor determinable depende de la metodología aplicada en los laboratorios encargados de realizar las pruebas oficiales de alcoholemia en los respectivos países, por lo que es mandatorio que el legislador al momento de establecer los valores máximos de alcohol en sangre permitidos para la conducción, realice una serie de análisis y consideraciones dentro de las cuales debe integrarse y valorarse la opinión técnicocientífica de las personas que trabajan en estos laboratorios y que son responsables de los análisis periciales; esto con el fin de que los límites fijados estén en consonancia con lo que es técnicamente posible de acuerdo a la casuística técnica de los laboratorios y sus metodologías analíticas.

En el caso particular de nuestro país y de acuerdo a los criterios técnicos científicos y al análisis comparativo de los límites de etanol en sangre para fines de conducción permitido en otros países; pudimos constatar que los valores asignados en nuestra legislación $(0,0007 \mathrm{~g} / \mathrm{L})$ difieren significativamente con lo que es posible diferenciar de manera confiable con la metodología analítica actualmente validada en nuestro país e incluso está muy alejada de lo establecido en otros países. Asimismo, difiere del umbral a partir del cual el alcohol fisiológicamente comienza a producir sus efectos.

El valor que se asignó en nuestra legislación de $0,0007 \mathrm{~g} / \mathrm{L}$, no es consecuente con el valor límite determinado por el Laboratorio. El valor que se considera como un área definida y un pico que no se debe discriminar es de $10 \mathrm{mg} / 100 \mathrm{~mL}$, equivalente a $0.1 \mathrm{~g} / \mathrm{L}$; este valor mínimo referido en nuestro laboratorio para considera la presencia del etanol en una muestra biológica en cambio el $0.07 \mathrm{mg} / 100 \mathrm{~mL}$ o su equivalente en gramos de $0,0007 \mathrm{~g} / \mathrm{L}$ que indica nuestra legislación como valor máximo permitido representa una cantidad, que tomada desde la analítica se interpreta como "no se detectó la presencia de etanol en sangre".

A manera ilustrativa se incluye la Figura 1, de un cromatograma que ejemplifica la altura de los picos. El cromatograma muestra tiempos de retención $\mathrm{SI}=2.65$, Etanol =1.66, (columnas RTX BAC PLUS 1 Y RTX BAC PLUS 1).

\section{CONCLUSIÓN}

Como se expuso en el presente trabajo, el análisis para la determinación de alcohol en sangre que se realiza en los laboratorios de Toxicología Forense de la Dirección de Medicina Forense de Tegucigalpa, ha sido validado y 
Figura1. Cromatografía doble detector FID con Automuestreador (HeadSpace)

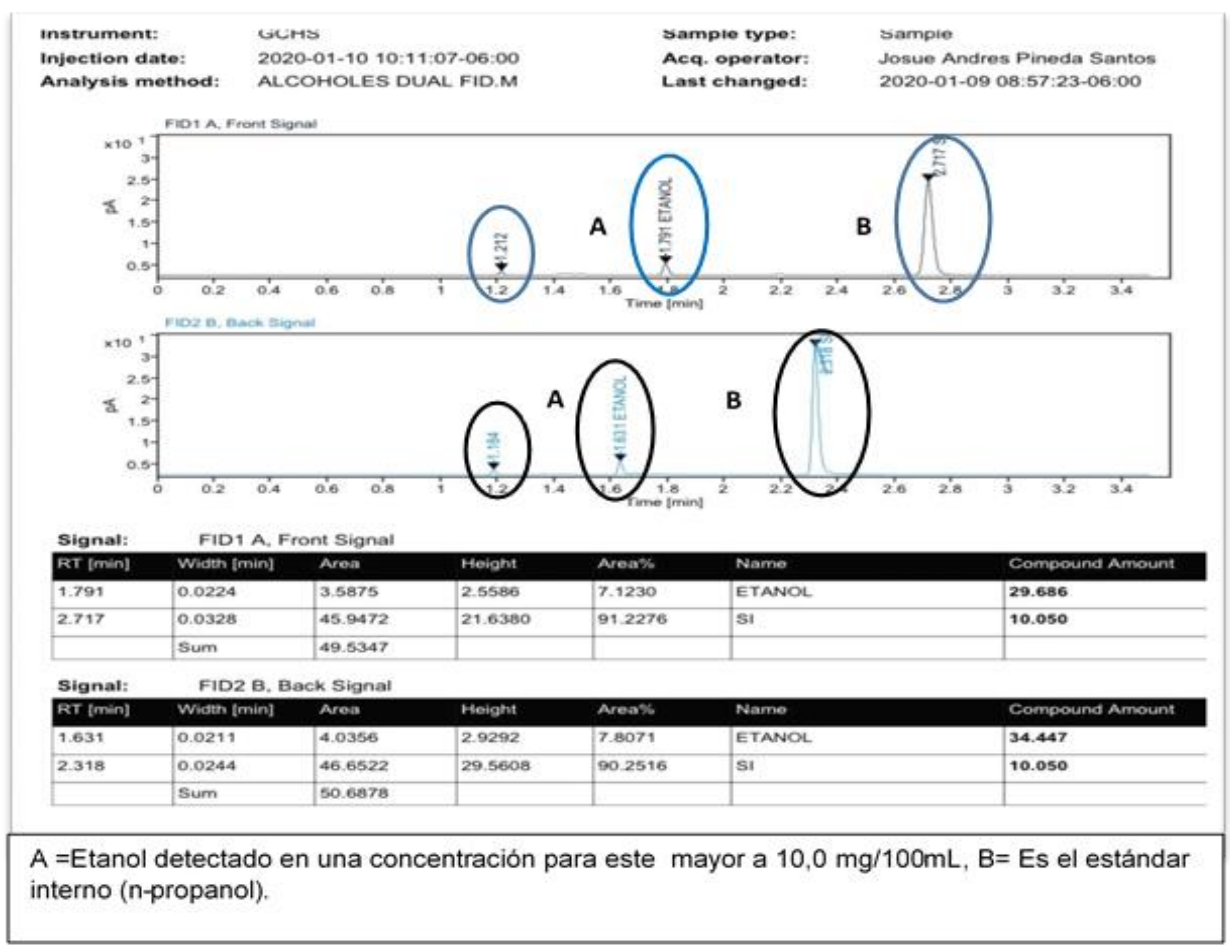

acreditado, y este proceso de validación ha permitido observar que el valor referido en la Ley de Penalización de la Embriaguez Habitual de Honduras, podría indicar un error en la aplicación de las unidades y de no ser un error, dicho valor no es significativo, desde la perspectiva técnica, en cuanto a la determinación y cuantificación del etanol en sangre por la metodología actualmente utilizada en los laboratorios.

La importancia del asesoramiento técnicocientífico para el uso adecuado de los valores y de las unidades es indispensable para una construcción y aplicación objetiva de nuestra legislación y es el punto de fusión de lo jurídico con lo científico.
El contar con un laboratorio acreditado como país es una oportunidad para el fortalecimiento de nuestras leyes, para no incurrir en errores y facilita que la prueba científica sea utilizada e interpretada de manera correcta.

\section{REFERENCIAS BIBLIOGRÁFICAS}

1.-Valencia Martin JL, González MJ, Galán I. Aspectos metodológicos en la medición del consumo de alcohol: la importancia de los patrones de consumo. Rev. Esp. Salud Pública [Internet]. 2014[citado 23 abril 2021];88(4):433-446. Disponible en: http://scielo.isciii.es/scielo.php?script=sci_artt ext\&pid=S1135-

\section{2\&lng=es\&nrm=iso>}

2.- Cayó JC, Tippetts AS, Voas RB. Accidentes de 
tráfico mortales que involucran a conductores ebrios: ¿qué hemos aprendido? Ann Adv Automot Med. 2009; 53:63-76.

3.-Widmark EMP. Principles and applications of medicolegal alcohol determination. Davis California: Biomedical Publications;1981.

4.-Desapriya EB, Iwase N, Brussoni M, Shimizu S, Belayneh TN. International policies on alcohol impaired driving: are legal blood alcohol concentration (BAC) limits in motorized countries compatible with the scientific evidence? Nihon Arukoru Yakubutsu Igakkai Zasshi. 2003;38(2):83-102.

5.- Molina Guerra AC. Niveles de alcohol en sangre detectados con mayor frecuencia en conductores que han sufrido accidentes de tránsito en el área metropolitana de Guatemala durante el año 2003. [Tesis en línea] Guatemala: Universidad de San Carlos ; 2004.[citado 23 mayo 2020].Disponible en: http://biblioteca.usac.edu.gt/tesis/06/06 2251 . $\mathrm{pdf}$

6.- Haghpanahan H, Lewsey J, Mackay DF, Mclntosh E, Pell J, Jones A, Fitzgerald N, Robinson M. An evaluation of the effects of lowering blood alcohol concentration limits for drivers on the rates of road traffic accidents and alcohol consumption: a natural experiment. Lancet. [Internet]. 2019[citado 23 mayo 2020];393(10169):321-329.

doi:
10.1016/S0140-6736(18)32850-2.

7.-Murillo W, Matamoros M, Alvarenga M. Una pareja explosiva: Alcohol y conducción. Rev cienc forenses Hondur. 2016;2(1):13-19.

8.- Consultor Jurídico Digital de Honduras. Ley de la penalización de la embriaguez habitual. Decreto 100-200. [Internet]. Tegucigalpa: Consultorio Jurídico; 2004. [citado 23 mayo 2020]. Disponible en: https://docs.bvsalud.org/leisref/2018/03/391/ alcohol-

leydelapenalizaciondelaembriaguezhabitual1.pdf

9.- Panel IV: Exámenes de Laboratorio y Alcoholemia. lus et Praxis [Internet].1996[citado 23 mayo 2020];2(1):7799. Disponible en:

https://www.redalyc.org/pdf/197/19720107.p df

10. Oficina de las Naciones Unidas Contra la Droga y el Delito. Directrices para la validación de métodos analíticos y la calibración del equipo utilizado para el análisis de drogas ilícitas en materiales incautados y especímenes biológicos. [Internet]. Nueva York: Naciones Unidas; 2010. [citado 23 mayo 2020]. Disponible en:

https://www.unodc.org/documents/scientific/ Validation Manual STNAR41 Ebook S.pdf 